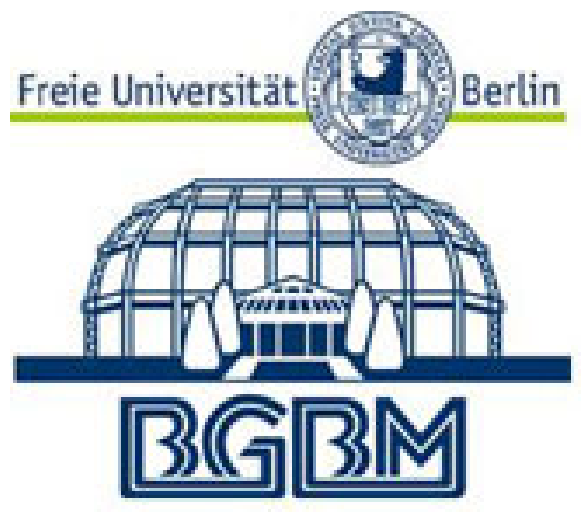

Die Kultur der von der botanischen Centralstelle in Berlin stammenden Nutzpflanzen in dem botanischen Garten von Viktoria-Kamerun

Author(s): Dr. Preuss

Source: Notizblatt des Königl. botanischen Gartens und Museums zu Berlin, Bd. 3, No. 29 (Jun. 30, 1902), pp. 198-213

Published by: Botanischer Garten und Botanisches Museum, Berlin-Dahlem

Stable URL: http://www.jstor.org/stable/3994048

Accessed: $15 / 06 / 2014$ 09:47

Your use of the JSTOR archive indicates your acceptance of the Terms \& Conditions of Use, available at http://www.jstor.org/page/info/about/policies/terms.jsp

JSTOR is a not-for-profit service that helps scholars, researchers, and students discover, use, and build upon a wide range of content in a trusted digital archive. We use information technology and tools to increase productivity and facilitate new forms of scholarship. For more information about JSTOR, please contact support@jstor.org. 
braucht, indem die letzteren einen Tag taber in solche Lösungen gelegt werden (sie ist sehr bitter), worauf die Haare sich ohne Mtihe mit der Hand ablösen lassen.

Der zweite Name des Baumes oder vielleicht auch der Beize ist in Hausa "medjĕmă."

In Dagomba wird die Rinde eines "kứcha" genannten Baumes als Mittel gegen Dysenterie angewandt, soll aber weniger wirksam sein.

Der bāgālŭa ist ziemlich häufig, wird sogar in und bei Städten z. B. Mangu wegen seiner Verwendung zur Gerberei angepflanzt.

\section{Die Kultur der yon der botanischen Centralstelle in Berlin stammenden Nutzpflanzen in dem botanischen Garten von Viktoria-Kamerun.}

Bericht des Dr. Preuss.

In dem Laufe der letzten drei Jahre ist die Thätigkeit der botanischen Centralstelle in Berlin fuir den Versuchsgarten in Viktoria ganz besonders fruchtbringend gewesen. Die Zahl der eingefubrten Arten ist auf mehr als 260 gestiegen, hat sich also seit $1898 \mathrm{mehr}$ als vervierfacht. Der starke Zuwachs wurde besonders ermöglicht durch das Zusammenwirken der botanischen Centralstelle mit dem kolonial-wirtschaftlichen Komitee und den von diesem ausgesandten Expeditionen. Die von letzteren eingesandten Sämereien oder lebenden Pflanzen wurden in den Gewächshäusern der Zentralstelle angezichtet oder eine Zeit lang gepflegt, um alsdann in Ward'schen Kästen in die Kolonieen versandt zu werden. Viktoria erhielt die grösste Anzahl von Pflanzen. Im folgenden will ich eine kurze Ubersicht tuber die Entwickelung der sämtlichen, durch die Zentralstelle dem botanischen Garten in Viktoria ubermittelten Arten geben.

\section{Genussmittel- und Nahrungsmittel - Pflanzen.}

Theobroma cacao. Die neu eingefthrten Varietäten dieser Art aus Guatemala und Mexiko gehören zu den wichtigsten Nutzpflanzen des botanischen Gartens, denn sie sollen zusammen mit den aus Venezuela direkt nach Viktoria eingefuhrten berühmten venezolanischen Spielarten die Grundlage zur Erzeugung erstklassigen Kakaos in Kamerun abgeben. Um für sie Platz zu schaffen, wurde ein Bestand älterer von Eingeborenen auf sehr gtinstigem Terrain am Limbeflusse gepflanzter 
Kakaobäume niedergelegt. Durch Ausfillen der sehr grossen Pflanzlöcher mit guter Erde und Düngerstoffen wurde den Pflänzlingen Ersatz für den schon etwas ausgesogenen Boden geliefert. Eine Anzabl derselben ging zwar ein und zweifellos, wie sich bei näherer Untersuchung herausstellte, infolge starker Verkrimmungen und Knickungen der Pfahlwurzeln, die bei dem Einsetzen der Bäumchen in die Pflanzkörbe entstanden sein muissen. Auch die übrigen zeigen meist keinen normalen Wuchs, haben jedoch die aussergewöhnlich starke Trockenzeit gut tiberstanden und werden sich wahrscheinlich gut weiter entwickeln.

Cola vera. Die Samen zu den vorhandenen Pflanzen stammen aus Jamaika, wo die Kolanuss vollständig akklimatisiert ist und vielfach angebaut wird. Der Umstand, dass die Samen dort oft grösser werden als in ihrer ursprünglichen Heimat in Afrika, war die Veranlassung zu der Überfihrung der akklimatisierten Jamaika-Kolanuss nach Kamerun, wo die Pflanzen vorläufig gut gedeihen.

Coffea arabica. Spielarten aus Mexiko, Costarica und Ecuador gedeihen vorläufig leidlich gut. Sie werden jedoch wahrscheinlich ebenso wie der bereits vorhanden gewesene arabische Kaffee im Alter von drei bis vier Jahren ein Opfer des westafrikanischen Kaffeekäfers, Monohammus sierricola werden. Weniger sicher scheint mir dieses zu sein bei der Coffea laurina, die in mehreren, sehr kräftigen Exemplaren vorhanden ist, wie denn auch z. B. die Coffea canephora vom Kuilu sowie zwei bei Viktoria wild wachsende Coffea-Arten in dem botanischen Garten noch nie von dem Käfer befallen worden sind.

Von dem "Bourbonkaffee" und zwar dem im Handel so hoch geschätzten „Bourbon pointu“ kam eine Quantität Saat an, jedoch keimte nicht ein einziges Korn, wahrscheinlich weil die Pergamenthaut von den Samen entfernt war.

Thea chinensis und var. assamica, welche in den ersten Jahren nur kümmerlich gediehen, scheinen sich allmählich zu akklimatisieren. Die Sträucher sehen gesund aus, sind freilich noch etwas sperrig, blithen und fruktifizieren aber reichlich. Es wird nunmehr eine Vermehrung durch Samen angestrebt.

Von den nach Buea verpflanzten Sträuchern sind die meisten bei Gelegenheit eines Baues vernichtet worden. Nur einige wenige sind noch am Leben, gedeihen aber in dem Gouvernementsgarten sehr gut.

Saccharum officinarum, Zuckerrohr, dem botanischen Garten von Kingston entstammend, entwickelt sich sehr kräftig. Da diese Varietät besonders reich an Zucker sein soll, so wird auf ihre Vermehrung Bedacht genommen, wenngleich vorläufig wenig Aussicht auf Bildung einer Zuckerrohrpflanzung in Kamerun vorhanden ist.

Erythroxylon novogranatense. Nur ein Strauch ist vorhanden. 
Erwähnen möchte ich an dieser Stelle, dass der Chemiker des botanischen Gartens, Herr Dr. Strunck, aus den Blättern der in Viktoria seit vielen Jahren kultivierten Kokaïnsträucher reines Kokaïn hergestellt hat, welches demnächst zur Prüfung nach Deutschland eingesendet werden wird. In den getrockneten, nach Europa gesandten Blättern konnte niemals das Alkaloid gefunden werden.

Paullinia cupana ist in zwei sehr jungen Pflanzen vorhanden.

\section{Gewtirzpflanzen.}

Vanilla planifolia. Eine Anzabl von Stecklingen, zu denen das Pflanzmaterial direkt aus den bertihmtesten Vanilledistrikten Mexikos bezogen war, sind an Hecken einer schmalblättrigen Varietät von Crescentia Cujete ausgepflanzt worden. Sie entwickeln sich vorläufig nur langsam, was vielleicht der abnorm starken Trockenzeit dieses Jahres zuzuschreiben ist. Vielleicht ist auch die Belaubung der Hecken den jungen Pflanzen zu dicht. Jedoch wird sich dieses Verbältnis voranssichtlich bald giinstiger gestalten.

Cinnamomum ceylanicum, Zimt. Von diesem Gewirz besteht nunmehr eine regelrechte kleine Pflanzung. Leider haben sich die Neger bei dem Schälen und noch mehr bei dem Schaben der Zimtrinde als so langsame Arbeiter erwiesen, dass mit ihnen an den erfolgreichen Betrieb einer Zimtplantage nicht zu denken ist. Es ist daber jetzt der Versuch gemacht worden, die Rinde in ungeschabtem Zustande nach Europa zu verschiffen, damit sie dort eventuell zur Destillation von Zimtöl benutzt werden kann.

Myristica fragrans, Muskatnuss. Die wenigen, durch die Centralstelle eingefubrten Bäumchen sind mit anderen, die direkt ans Trinidad nach Viktoria gelangten, in einem kleinen geschlossenen Bestande am Fusse des sogenannten Kaffeeberges ausgepflanzt worden. Als Schattenspender dienen ein Pithecolobium und Schizolobium excelsum. Übrigens haben die aus Singapore und São Thomé in den Jahren 1893 und 1894 eingefuhrten Muskatnussbäumchen bereits die Tragbarkeit erreicht, und es hat sich herausgestellt, dass nicht nur die kleine, längliche, sondern auch die grosse, runde, wertvollere Varietät vorhanden ist. Letztere hat die starke Trockenzeit weit besser überstanden als erstere, deren Frichte einschrumpften und nicht zur vollen Entwickelung gelangten. Als Schattenbäume dient hier Canarium ceylanicum. Das Kamerungebirge besitzt in gewissen Höhenlagen sehr ausgedehnte, für die Kultur der Muskatnuss vorzüglich geeignete Landstrecken.

Elettaria cardamomum. Kardamom. Aus den eingesandteu Samen sind eine ganze Anzahl kräftiger Pflänzlinge gezüchtet worden. 
Ob sich mit dieser Saat ginstigere Resultate erzielen lassen werden als mit der im Jahre 1893 aus Ceylon bezogenen, bleibt abzuwarten.

Pimenta officinalis, Piment, ist nur in einem, allerdings sehr gut gedeihenden Exemplare vorhanden.

Piper nigrum, schwarzer Pfeffer, liefert nach wie vor Frïchte von hervorragender Schärfe und vorzüglichem Aroma, aber leider nicht reichlich genug. Die fribhere Anpflanzung hat zum grössten Teile dem neu eingefuhrten Venezuelakakao Platz machen müssen. Es sind nur so viele Sträucher übrig geblieben, dass alle Jahre einige Pfund geernet werden können und dass jeder Zeit Pflanzmaterial zur Abgabe an die Pflanzungen vorhanden ist (24).

Piper angustifolium, $P$. betle und $P$. officinarum gedeihen gut. Zingiber Clarkei ist erst kiirzlich eingeftihrt worden und steht gut.

\section{Essbare Fruchtarten.}

Die Obstarten und solche Pflanzen, welche essbare Frtichte liefern, nehmen in dem botanischen Garten einen bedeutenden Raum ein. Ihre Zahl reicht an 90 heran, und von diesen sind nicht weniger als 49 durch Vermittelung der botanischen Centralstelle bierhergelangt. Mit nur sehr wenigen Ausnahmen behagt ihnen das Klima von Viktoria gut, und wenn einige, wie Nephelium Longana und Flacourtia inermis trotz ibrer guten vegetativen Entwickelung noch keine Frïchte ansetzen, so ist dieses wohl als eine Eigentimlichkeit der Arten anzusehen.

Unter denen, die in dem letzten Jahre zur Fruchtentwickelung gelangt sind, ist vor allen anderen zu nennen Spondias dulcis $={ }_{n}$ Cajamanga " der Portugiesen, "Pomme cythère" der Franzosen, „Waterplum" der Engländer. Vierjährige Bäume dieser Art waren so mit Frtichten uiberladen, dass viele Äste brachen. Die Reifezeit dauerte ununterbrochen länger als ein halbes Jahr, vom Mai bis zum Dezember. Im Februar standen die Bäume bereits wieder in Blüte, nachdem kurz vorher der Abfall der Blätter stattgefunden hatte. Die sehr erfrischenden Fribchte, welche bisweilen die Grösse einer Faust erreichten, erfreuten sich bei Europäern wie bei Schwarzen einer gleich grossen Beliebtheit. Samen davon wurden uiberall im Schutzgebiete verbreitet und auch in beträchtlicher Anzahl der botanischen Centralstelle übersandt.

Spondias Mombin Jacq., obgleich in demselben Alter wie S. dulcis hat noch nicht gebliht. Die Früchte dieser Art, obgleich von sehr schönem Aroma, erfreuen sich übrigens wegen ihrer starken Säure und wegen eines Kratzens in dem Halse, den ihr Genuss erzeugt, nur einer geringen Beliebtheit.

Garcinia xanthochymus, obgleich erst kaum $3 \mathrm{~m}$ hoch, setzte gleich- 
alls zum ersten Male Frtichte an. Letztere entbehren leider des Aromas, haben aber eine erfrischende Säure.

Anona cherimolia lieferte zum ersten Male zwei kleine Früchte. Dieselben erinnerten freilich im Geschmack nur wenig an diejenigen im Hochlande von Venezuela oder auf der Insel Madeira. Das heisse, feuchte Küstenklima von Viktoria bekommt ihnen nicht gut. Letzteres gilt in gleichem Masse fur Eriobotrya japonica, welche jetzt auch bereits öfters Früchte trägt, die aber bei weitem nicht so erfrischend und wohlschmeckend sind wie diejenigen in Madeira.

Anona squamosa dagegen liefert einen grossen Teil des Jahres hindurch sehr gute Früchte, und die verschiedenen neu eingefubrten Varietäten der A. reticulata ans Salvador, Mexiko, Nicaragua und Guatemala, ferner Anona manirote aus Venezuela und Anona spec. aus Surinam nehmen eine vortreffliche Entwickelung. Zahlreiche Exemplare aller dieser Arten sind in dem Schutzgebiete verbreitet worden.

Achras Sapota trägt gleichfalls bereits Früchte, und die verschiedenen, neu eingeführten Varietäten gedeihen gut.

Dasselbe gilt für Chrysophyllum Cainito, welches zum ersten Male sehr grosse, grïne Frlichte getragen hat, ferner für Ch. monopyrenum mit kleinen, einsamigen Frichten und andere Chrysophyllum-Arten.

Carica papaya in den aus Zentralamerika eingefthrten Varietäten erregt durch die kolossalen Frïchte allgemeine Aufmerksamkeit. Besonders die Eingeborenen bemïhen sich diese Varietäten, welche den afrikanischen an Wohlgeschmack weit tiberlegen sind, anzupflanzen.

Averrhoa Carambola, Jambosa vulgaris, Blighia sapida, Artocarpus integrifolia und Psidium guayava fruktifizieren reichlich. Leider sind die Schwarzen an den Genuss des Arillus von Blighia sapida nicht zu gewöhnen, ebensowenig geniessen sie das Fruchtfleisch oder die Samen von Artocarpus integrifolia.

Von den neu eingefuhrten Arten sind besonders erwähnenswert Garcinia mangostana, die freilich schon in älteren, jetzt bis $2 \mathrm{~m}$ hohen Bäumchen vorhanden ist, ferner Durio zibethinus, eine ganze Anzahl veredelter Mangosorten, Syzygium jambolanum, Citrus decumana, Citrus aurantium und andere Citrus - Arten, Persea gratissima in den verschiedensten Varietäten, edle Ananas-Arten, die Martinique-Banane, Meliococca bijuga, Monstera deliciosa, Eugenia malaccensis und $\boldsymbol{E}$. javanica, Chrysobalanus icaco, Inga edulis und Inga spec., eine Lucuma-Art, "Ingerto" genannt, drei Passiflora-Arten, Tamarindus indica, eine Wallnuss aus Mexiko, die Tempisque-Pflaume aus Guatemala, Mammea americana in verschiedenen guten Varietäten, Prunus capollin, Casimiroa edulis, Aegle marmelos, Artocarpus lacoocha, Catha edulis, Caryocar nuciferum, Flacourtia 
cataphracta, Punica granatum, Strychnos rouhamon, Zizyphus jujuba, Morinda citrifolia, Castanospermum australe und andere mehr.

Von anderen Arten, die entweder als Gemiise oder als Viehfutter Verwendung finden, sind noch zu erwähnen Pachyrhizus angulatus, der sehr reichlich Früchte trägt, Brosimum alicastrum und Sarcocephalus esculentus.

\section{Medizinalpflanzen.}

Unter ihnen scheint die grösste Zukunft in Kamerum zu baben Myroxylon Pereirae, der Perubalsambaum. Schon seit zwölf Jahren befinden sich vier Bäume davon in dem botanischen Garten. Neuerdings aber sind aus Salvador Samen eingefuhrt worden, aus denen mehrere Hunderte von Bäumen angezlichtet worden sind. Ein kleiner Teil davon ist an die verschiedenen Pflanzungen verteilt worden; die uibrigen sind an drei Stellen in dem botanischen Garten, teils an Hängen teils im Flachlande in kleinen Beständen ausgepflanzt worden in Entfernungen von 6 bis $8 \mathrm{~m}$. An zwei Stellen sind Bäume von Berrya amomilla in Entfernungen von 2 bis $3 \mathrm{~m}$ zwischen die Balsambäume gepflanzt, um sie zu geradem Wuchse und zur Bildung schöner Stämme zu zwingen. Die letzteren gedeihen durchweg sehr gut, und selbst an den steilen, trockenen Hängen hat ihnen die sebr ausgesprochene Trockenzeit nichts anhaben können.

In grösserem Masstabe ist die Kultur des Balsambaumes allerdings nur auf der Moliwepflanzung in Angriff genommen worden.

Toluifera balsamum, Tolubalsam, ist nur in wenigen jungen Exemplaren vorhanden.

Croton tiglium. Diese Art war, wie schon in einem fruheren Berichte erwähnt worden ist, reichlich vermehrt worden. Es wurde alsdann Ende 1901 eine Quantität von etwas weniger als einem Zentner zum Verkauf nach Hamburg gesandt und zu dem Preise von $45 \mathrm{M}$. per 100 Kilo verkauft. Die Nachfrage nach Crotonsamen ist indessen in den letzten Jahren so stark gesunken, dass von einer weiteren Anpflanzung des Strauches in grösserem Massstabe abgesehen werden soll. Die vorhandenen Sträucher werden vorläufig geschont, da sie als Stutzen fiir Strophanthus gratus dienen.

Cinchona calisaya. Eine Anzahl von Pflänzlingen langte in leidlich gutem Zustande in Viktoria an und wurde zur Anpflanzung im Gebirge nach Buea gesandt.

Cinnamomum camphora. Die vorhandenen 24 Bäume entwickeln sich gut und sind jetzt im Durchschnitt $4 \mathrm{~m}$ hoch. Um ihnen eine gute Form zu geben, muss man sie häufig beschneiden, wogegen sie glïcklicherweise nicht empfindlich sind. 
Brucea antidysenterica wurde in einem ans Jamaika stammenden Exemplare dem botanischen Garten tibersandt, in welchem sich aber seit Jahren mehrere alte Sträucher dieser Art, welche in dem Kamerungebirge wild wächst, in Kultur befinden. Dieser Art soll ebenso wie anderen Simarubaceen erhöhte Aufmerksamkeit geschenkt werden wegen der ihrer Rinde innewohnenden Heilkraft gegen Dysenterie. Einige wildwachsende Pflanzen sind aus dem Kamerungebirge in den Gouvernementsgarten in Buea tibergefuhrt worden.

Quassia amara, Bitterholz, soll eine ähnliche Heilkraft besitzen wie vorgenannte Brucea. Sie ist durch ein älteres, bereits blibendes, und zwei junge Exemplare im Garten vertreten.

Marsdenia condurango hat sich an mehreren Stellen im Garten, sowohl epiphytisch auf Ficus religiosa und Spondias dulcis, als auch im Erdboden wurzelnd angesiedelt und fruktifiziert reichlich. Die Stämme bleiben jedoch sehr diun, und es ist nicht abzusehen, wieviel davon nötig sein wird, um ein Kilo Rinde zu produzieren.

Smilax medica, die echte Zarzaparilla, aus Jalapa in Mexiko stammend, ist in einigen sehr langsam wachsenden jungen Exemplaren vorhanden. Den Blättern nach zu urteilen ist diese Art nicht identisch mit der vor einigen Jahren unter denselben Namen von Berlin nach Viktoria gesandten Art, obgleich letztere, die sehr üppig gedeiht, auch an der Erdoberfläche die zahlreichen, biischeligen, der Zarzaparilla eigentiumlichen Wurzeln bildet, welche in den Handel kommen.

Strophanthus-Arten. Zu dem fruilier von der botanischen Zentralstelle eingeschickten Strophanthus caudatus sind neuerdings hinzugetreten: Strophanthus hispidus in mehreren Exemplaren, S. "regalis" und S. "Stanleyanus". S. caudatus bliuht reichlich, setzt aber nie Frucht an. Von den älteren, im botanischen Garten vorhandenen Arten habeı S. hispidus, S. gratus, $S$. „kombe" und eine in Kamerun wildwachsende Art zum Teil sehr schön geblïht, aber nur $S$. gratus hat einige wenige Frtichte entwickelt.

Strychnos nux vomica hat Stämme von 5 bis $6 \mathrm{~m}$ Länge, hat jedoch noch nie gebliiht.

Uragoga ipecacuanha ist vollständig verschwunden.

Curcuma longa ist stark vermehrt worden, desgleichen die Kaempferia galanga.

Pilocarpus racemosus ist in mehreren, Anamirta Cocculus in einem jungen Exemplare vorhanden.

Dipteryx odorata, die Tonkabohne, ist vertreten durch zwei junge, aber kräftige Pflanzen, denen besondere Aufmerksamkeit geschenkt wird, da diese Art ausser dem Nutzen, den sie durch ihre Samen bringt, sich auch vielleicht zum Schattenbaum für Kakao eignet. 


\section{5. Ölpflanzen.}

Aleurites moluccana bildet jetzt bereits stattliche Bäume, welche reichlich Frucht tragen. Aus den angenehm nussartig schmeckenden Samen, welche leider eine etwas abfuhrende Wirkung haben, bereitet zur Zeit der Chemiker des Gartens, Herr Dr. Strunck, ein öl, iiber dessen Eigenschaften und Wert genauere Ermittelungen angestellt werden werden.

Illipe latifolia blihht regelmässig, jedoch tragen die kräftigen, dicht belaubten Bäume trotz ihres gesunden Aussehens niemals Frucht.

Bassia longifolia ist zu einem mehr als $7 \mathrm{~m}$ hohen Baume mit sehr dünner, schmaler Krone herangewachsen.

\section{Farbpfianzen.}

Coulteria tinctoria. Auch die beiden letzten Pflanzen, die längere Zeit kummerlich vegetierten, sind eingegangen.

Garcinia cochinchinensis bildet jetzt zwei kleine Bäumchen von $3 \mathrm{~m}$ Höhe, G. xanthochymus hat bereits Fribhte getragen.

Garcinia morella, G. Loureiri und andere Farbstoffe liefernde GarciniaArten sind nur in ganz jungen Exemplaren vorhanden.

Mallotus philippinensis; ein kräftiger Strauch.

Caesalpinia coriaria entwickelt sich zu kleinen Bäumen mit lang ubberlängenden Ästen. bliiht.

Caesalpinia sepiaria hat in diesem Jahre zum ersten Male ge-

Caesalpinia sappan, welche früher an den Standörtern nahe der See stets einging, gedeiht an einem feuchten, von der See abgelegenen Platze vorztiglich. Die stacheligen Stämme geben abwechselnd mit Fourcroya gigantea einen selrr guten Grenzzaun ab. Frtichte sind in Menge vorhanden.

Haematoxylon campechianum bildet niedrige, knorrige Stämme und hat bereits geblitht.

\section{Kantschukpfianzen.}

Die Zahl der Kautschukpflanzen, welche neuerdings in den botanischen Garten von Viktoria ubergeftihrt worden sind, ist sehr beträchtlich sowohl in Bezug auf die Zahl der Arten als anch auf diejenige der Individuen. $\mathrm{Zu}$ nennen sind folgende Arten:

Castilloa elastica, Sapium utile, Mascarenhasia elastica, Hevea Sieberi, "Ficus elastica" aus Indien und "Ficus elastica" aus Natal, beide von einander selir verschieden, Forsteronia floribunda, Cryptostegia grandiflora und zwei unbestimmte Kautschuklianen aus Madagaskar, deren eine zu den Apocynaceen die andere zu den Asclepiadaceen gehört. 
Castilloa elastica ist in mehreren hundert Pflanzen eingeftihrt worden; davon wurden tiber 300 in dem Schutzgebiete verteilt. Die Pflanzen stammen aus verschiedenen Teilen von Suid- und Zentralamerika, wie Britisch-Guyana, Ecuador, Nicaragua, Costarica, Guatemala und auch aus Westindien, wie Jamaika und Trinidad. Es scheinen mehrere verschiedene Varietäten vorhanden zu sein, aber nur ein kleiner Teil gehört zu der von Koschny in Costarica als Castilloa alba bezeichneten und von ihm eingesandten Varietät. Die fuinf ältesten Bäume hatteu im Alter von drei Jahren schon eine recht stattliche Grösse erreicht, da wurden vier von ihnen plötzlich von Käferlarven befallen, welche in dem Stamme selbst Gänge bohrten und von dort aus die Rinde zerfrassen. Die Oberhaut der Rinde wurde dabei verschont, so dass man nicht sogleich auf die Schädlinge aufmerksam wurde. So kam es, dass ein Baum völlig abstarb. Aus den tibrigen drei sind bis jetzt fünf Käferlarven entfernt worden, welche dicht unter der Oberhaut der Rinde in letzterer selbst sich befanden; aber die Verletzungen der Bäume sind sehr schwer, und es ist zweifelhaft ob sie am Leben bleiben werden, zumal sie an den zerfressenen Stellen leicht von den Tornados geknickt werden können.

Die tibrigen Castilloa sind teils in Abständen von $15 \times 10 \mathrm{~m}$ und von $4 \times 10 \mathrm{~m}$ zwischen Kakao, teils in Abständen von $6 \times 6 \mathrm{~m}$ zwischen Kardamom, teils auch in Abständen von $6 \times 3$ oder $6 \times 6 \mathrm{~m}$ in kleinen geschlossenen Beständen ausgepflanzt werden. Wenn ich auch die Castilloa nicht für einen gecigneten Schattenbaum für Kakao halte, so halte ich es doch für eine Aufgabe der Versuchspflanzung in Viktoria, dieses an Beispielen zu zeigen und festzustellen, wie gross der Schaden der Castilloa im Verhältnisse zu ihrem Nutzen ist. Und zwar sind die betreffenden Versuche in ganz gleicher Weise auch mit Kickxia elastica angestellt worden, so dass man bald sehen wird, welchem von beiden Bäumen der Vorzug zu geben ist.

Sapium utile ist nur in fünf Exemplaren vorbanden. Einige Pflanzen wurden ebenso wie viele Kickxia elastica von Schnecken durch Abfressen der Rinde während der Nacht getötet. Die übrig gebliebenen wachsen schnell, und das stärkste Bäumchen hat eine Höhe von 1,50 m erreicht.

Mascarenhasia elastica ist in 16 Exemplaren vorhanden, deren beste bis $2 \mathrm{~m}$ hoch sind. Sie entwickelt bereits reichlich Bliten; ich weiss nicht, ob dieses eine normale Erscheinung bei ihr ist. Sie ist in Gesellschaft mit Hevea brasiliensis und Castilloa elastica angepflanzt, um Vergleiche zu ermöglichen.

Hevea brasiliensis Die im Jahre 1896 aus Berlin gekommenen Bäumchen haben 1901 bereits Früchte getragen, welche zur Anzucht von Sämlingen benutzt wurden. Eine Anzahl derselben ist an die 
Pflanzungen in dem Schutzgebiete verteilt worden, einige sind auch noch in dem botanischen Garten ausgepflanzt worden, der jetzt im ganzen 68 Hevea brasiliensis besitzt, wovon sechs bereits zehn Jahre alt sind. Letztere dienen als Schattenbäume für Kakao, wozu sie sich sehr gut zu eignen scheinen. Leider geben sie selbst in diesem Alter nur wenig Kautschuk.

Hevea Sieberi. Unter diesem Namen sind neuerdings zwei junge Pflanzen eingefuihrt worden, welche der Hevea brasiliensis sehr ähnlich sehen.

Ficus elastica. Die eingesandten Samen keimten nur zum allergeringsten Teile. Der Garten besitzt jetzt 41 Pflänzlinge. Unter demselben Namen gelangte eine Anzahl Pflanzen von der Zentralstelle nach Viktoria, welche jedenfalls etwas anderes sind als Ficus elastica. Als Ursprungsland ist Natal angegeben. Sie entwickeln eich sehr gut. Ihre Zahl beträgt zehn.

Forsteronia floribunda. Von dieser aus Westindien stammenden Kautschukliane erhielt der botanische Garten sowohl Samen als auch Pflanzen. Dieselben ähneln so sehr der folgenden Art, dass man sie nicht von ihr unterscheiden kann.

Cryptostegia grandiflora. Ausser den Pflanzen, welche aus dem von der Zentralstelle stammenden Samen geztichtet worden sind, besitzt der Garten noch zwei Exemplare, welche aus dem botanischen Garten von Grenada stammen, aber auch diese sehen der Forsteronia floribunda zum Verwechseln ähnlich. Eine sichere Scheidung wird wahrscheinlich erst durch die Blitte ermöglicht werden.

Landolphia sp? und eine zu den Asclepiadaceen gehörige Kautschukliane aus Madagaskar haben die Trockenzeit leidlich gut uiberstanden und düften sich gut weiter entwickeln.

\section{Guttaperchapflanzen.}

Von Guttapercha liefernden Arten besitzt der botanische Garten vorläufig nur sieben kleine Pflanzen von Payena Leerii. Das einzige von der Zentralstelle eingesandte Exemplar von Palaquium gutta ist eingegangen.

Mimusops balata, die Stammpflanze des Balata-Guttapercha, ist in einem Exemplar von der Zentralstelle eingesandt worden, welches aber sicherlich eine andere Art darstellt als diejenige Pflanze, die ich unter demselben Namen aus Britisch-Guyana in sechs Exemplaren erhalten habe. Dagegen gleicht sie vollständig derjenigen Art, die seit elf Jahren unter der Bezeichnung Sapota Mülleri in Viktoria kultiviert wird. Interessant wird es sein, die beiden Arten nebeneinander zu beobachten: 
Tabernaemontana Donnell-Smithii, deren aus den Fribchten gewonnene Milch eine Art Guttapercha liefert, ist in grösserer Anzahl aus Samen angezüchtet worden. Die Pflanzen wurden in Abständen von $6 \times 6 \mathrm{~m}$ in einem kleinen Bestande ausgepflanzt. Sie hatten während der Regenzeit ausserordentlich stark an Schneckenfrass, in der Trockenzeit dagegen an Raupenfrass zu leiden und sind deshalb stark in der Entwickelung zurtickgeblieben. Das stärkste Exemplar steht jetzt in Blite.

Zu erwähnen wäre im Anschlusse an die Kautschuk- und Guttaperchapflanzen noch

Galactodendron utile, der Kuhbaum von Venezuela. Derselbe ist in nur einem Exemplar vorhanden, welches sehr langsam wächst. Die Küstenatmosphäre von Viktoria scheint der Hochlandspflanze nicht zu behagen.

\section{Faserpfianzen.}

Corchorus capsularis var. attariya Schon frïher ist berichtet worden, dass die Jutepflanze, wenigstens die vorliegende Spielart, sich zu stark verästelt und zu niedrig bleibt. Die fortgesetzten Versuche haben stets dasselbe Resultat ergeben.

Boehmeria nivea. Aus Samen der Ramie, die einer besonders guten indischen Varietät entnommen worden sein sollen, ist eine kleine Anzahl von Pflanzen gezilchtet worden. - Von der in dem botanischen Garten fruher kultivierten Varietät sind beträchtliche Quantitäten von Saatwurzeln an einzelne Plantagen und Gesellschaften abgegeben worden. Für diejenigen Leute, die sich mit dem Gedanken der Ramiegrosskultur in Kamerun abgeben, sei hier bemerkt, dass die Ramie auf dem Versuchsfelde am 5. November 1901 geschnitten wurde und dass sie während der Trockenzeit bis zum Ende Februar 1902 nicht einmal die Durchschnittshöhe von $1 \mathrm{~m}$ erreichte. Auf dem hilgeligen Teile des Versuchsfeldes blieb sie nur einen halben Meter hoch.

Baumwolle in zwei aus Stidamerika stammenden Varietäten fruktifizierte reichlich. Die im Juli zur Aussaat gelangten Sträucher reiften ihre Kapseln während der Trockenzeit schön aus, und es konnte eine gute Probe gesammelt werden. So stark ausgeprägte Trockenzeiten wie die diesmalige sind allerdings in Kamerun nicht die Regel.

Bombax malabaricum und Ochroma lagopus sind in einigen Exemplaren aus Samen geziichtet worden.

Phormium tenax, Musa textilis (?) und Pandanus utilis sind in wenigen, gut gedeihenden Exemplaren vorhanden.

Von besonderer Wichtigkeit sind die aus Sijd- und Zentralamerika eingeftihrten Agave-Sorten, besonders die den Henequen liefernde Sisal- 
agave, die Cabnyaagave ans Zentralamerika und diejenige aus Chile, ferner die Ixtle-, Lechuguilla- und Pulqueagave aus Mexiko, welche sämtlich mehr oder weniger brauchbare Fasern liefern.

Bromelia sp? aus Nicaragua liefert nicht nur eine gute Faser, sondern dient auch als Wegeeinfassung. Die Früchte, „Piñuela dulce" genannt, werden gegessen.

\section{Schattenbäume.}

Unter den seit mehreren Jahren kultivierten Schattenbäumen haben sich bis jetzt als die besten herausgestellt: Erythrina lithosperma, welche wohl mit $E$. indica, dem "Dadap“, identisch ist, ferner E. umbrosa, welche mit $E$. glauca, der "Koffiemama" von Surinam, und dem "Bucare pionio" von Venezuela und dem "Bucare" von Trinidad uibereinstimmt, ferner Albizzia stipulata, A. lebbeck in einer bestimmten Varietät, Pithecolobium Saman und Canarium zeylanicum. - Albizzia moluccana in erwachsenem Zustande erweist sich als weit widerstandsfähiger gegen Winde etc. als im Jugendzustande und erscheint mir jetzt weit weniger verwerflich als früher.

Artocarpus integrifolia, Acrocarpus fraxinifolia und Erythrina corallodendron werden nicht melir als Schattenbäume betrachtet. Letztere wird noch als Stützbaum für Vanille versucht.

Erythrina lithosperma und E. umbrosa lieferten Material zu mehreren tausend Stecklingen, welche an die Kakaopflanzungen abgegeben wurden, ausserdem haben sie bereits gebliht und von $E$. lithosperma ist eine ganze Anzahl von Sämlingen geziichtet worden. Albizzia stipulata lieferte viele tausend Samen und $A$. Lebbeck etwa ein Tausend. Vou Pithecolobium Saman wurden Pflanzen und Samen an die Plantagen abgegeben.

Von den neu eingefürten Schattenbäumen sind besonders zu nennen Erythrina glauca, E. micropteryx, der "Bucare ananco" von Venezuela und "Anauco" von Trinidad, ferner E. velutina und drei andere Erythrina-Arten, vou denen der "Pericocco" von Venezuela und der "Pito" von Salvador zusammen mit $E$. lithosperma auch als Stiitzbäume für Vanille verwendet werden. Alle Erythrina-Arten werden leider durch Raupen von Mikrolepidopteren stark geschädigt, welche die $\mathrm{Z}_{\text {weigspitzen }}$ ausfressen.

Ferner erwähne ich Gliricidia sepium, die "Madre de Cacao" von Zentralamerika, Canarium commune, Inga edulis und Inga sp? sowie die unter den spanischen Namen "Castaneo", "Tepe-Aguacate" und "Guachapeli bekannten Bäume, welche erst noch wissenschaftlich bestimmt werden sollen.

Als Stutzbaum ftir Vanille und eventuell Pteffer ist noch Crescentia trifoliata neben $C$. cujete, Gliricidia sepium und den genannten, stachel- 
losen Erythrina-Arten in Gebrauch genommen worden. Crescentia cucurbitacea eignet sich nicht gut zum Stiitzbaume.

Der botanische Garten in Viktoria befindet sich somit in dem Besitze der meisten guten Schattenbäume der Welt und wird in wenigen Jahren im stande sein, etwa neu sich bildenden Pflanzungen die sämtlichen erforderlichen Schattenbäume zu liefern, so dass keine Urwaldbäume mehr geschont zu werden brauchen.

\section{Nutzholger.}

Tectona grandis, Teakholz. Der älteste, im Jahre 1897 von der Zentralstelle nach Viktoria gesandte Baum hat eine stattliche Grösse erreicht und fruktifiziert reichlich. Er hat bei der letzten Blute mehr als 1500 gute Samen geliefert. Ausserdem sind aus den von der Zentralstelle eingesandten Samen über 2000 Pflanzen geziichtet worden, welche in der Regenzeit an den Regierungswegen ausgepflanzt werden sollen. In dem botanischen Garten soll ein kleiner, geschlossener Bestand davon angelegt werden. Einzelne Bäumchen sind an verschiedene Pflanzungen zur Verteilung gelangt.

Cedrela odorata, Ceder, ist nur in wenigen Exemplaren vorhanden, die aber dem Teakholz an Schnelligkeit des Wachstums nicht nachstehen. Der älteste, vier Jahre alte Baum ist bereits $12 \mathrm{~m}$ hoch und ditfte in nicht zu ferner Zeit zur Blüte kommen.

Eine zweite, nahe verwandte Art, die freilich nicht ganz so gutes Holz liefert, ist in einer ganzen Anzahl von jungen, einjährigen Bäumen an Wegen und in einem kleinen Bestande auf dem Gipfel des Kaffeeberges angepflanzt worden. Auch diese Art hatte sehr stark unter Schneckenfrass zu leiden.

Swietenia mahagoni wächst bedeutend langsamer als Teak und Ceder, besonders dort, wo er beschattet ist. Der älteste, etwa vier Jahre alte Baum ist erst gegen $4 \mathrm{~m}$ hoch. Er wird stark von Ameisen belästigt, welche starke Wucherungen der Rinde und den Ausfluss eines Harzes veranlasselı. - Zahlreiche Bäumchen sind an den Wegen im Garten ausgepflanzt worden. Sie gedeihen gut, auch an ganz trockenen Hängen, ebenso wie

Swietenia bijuga aus Salvador, welche gleichfalls in zahlreichen Exemplaren ausgepflanzt worden ist. Letztere Art wächst bedeutend schneller als erstere und scheint Trockenheit noch besser zu vertragen als jene.

Calophyllum inophyllum bildet schon hübsche Alleen und einen kleinen Bestand am Osthange des Kaffeeberges. Er ist in grosser Menge im Schutzgebiete verbreitet worden. Diese Art ist anffallend stark heliotropisch; jeder benachbarte Baum oder Bambusstrauch, ja 
jede Bananenstaude ist im stande, den Stämmen eine schiefe Richtung zu geben, was bei der Anlage von Alleen bisweilen recht störend ist.

Mesua ferrea, Eisenholz. Das einzige von sechs ubrig gebliebene Bäumchen ist jetzt zu einem mehr als $2 \mathrm{~m}$ hohen Stämmchen herangewachsen, welches die ersten Bltitenknospen angesetzt hat. Die Art wird boffentlich dem Garten erhalten bleiben.

Dalbergia sissoa ist in einer kleinen Anzahl von Bäumen von 3 bis $6 \mathrm{~m}$ Höhe vorhanden.

Michelia champaca, die nicht nur Nutzholz sondern auch ein gutes Parftim liefert, ist in drei Stämmen vorhanden, welche kerzengerade bis zu $25 \mathrm{~m}$ Höhe emporsteigen. Sie sind jetzt in dem unteren Teile astlos und tragen eine schmale, pyramidenförmige Krone. Seit einem Jahre tragen sie Fritichte.

Cordia subcordata. Vier Stämmchen von 2 bis $3 \mathrm{~m}$ Höhe.

Haematoxylon campechianum. Zwei Stämrne von unschönem Wuchse. Neu eingefuhrt und in jungen, etwa $1 \frac{1}{2}$ jährigen Bäumchen sind folgeude Arten: Bocagea virgata, das sogenannte "Lancesparwood ${ }^{4}$ aus Jamaika, Diospyros discolor und D. ebenaster, Jacaranda filicifolia, Podocarpus elata, Piscidia erythrina, Hymenaea courbaril, Guajacum sanctum, dessen Kultur trotz friherer Misserfolge wiederum versucht werden soll, Tabebuya serratifolia, Nectandra gerascanthus $=$ "Volador ${ }^{\text {" von Guate- }}$ mala, Spondias axillaris, Pterocarpus draco, Intsia amboinensis und andere mebr.

Stadmannia australis ist eingegangen.

Von Bäumen, die wenig oder gar keinen Nutzen haben, sondern nur als Alleebäume etc. angepflanzt werden, sind zu nennen Ficus lucida, F. altissima, Sterculia foetida, St. elastica, Parkia biglandulosa, Parmentiera cerifera, Grevillea robusta, Cassia nodosa, C. grandis, C. brasiliana, C. glauca, Pithecolobium unguis cati, Semecarpus anacardium, Schizolobium excelsum, Couroupita guyanensis etc.

\section{Bambusarten.}

Bambusa arundinacea. Von den beiden Pflanzen, welche im Jahre 1898 durch Hochwasser fortgerissen schieneu, hat sich eine wieder eingefunden und ist zu einem starken Busche mit $10 \mathrm{~m}$ langen Halmen herangewachsen. Zahlreiche Pflanzen dieser Art wurden aus Samen gezüchtet, welche von Indien kamen.

B. vulgaris, aus Jamaika übergefuihrt, gedeiht sehr gut, ist vermehrt und am Ufer des Limbeflusses ausgepflanzt worden. Vergleiche ergaben, dass der in Kamerun seit Menschengedenken kultivierte und vielleicht sogar daselbst wilde Bambus der Bambusa vulgaris sehr nahe steht. 
B. regia bildet ausserordentlich vielstämmige und dichte Buische von schöner Form.

Dendrocalamus strictus. Die unter diesem Namen vor mehreren Jahren durch die Zentralstelle eingesandte Art bildet stets nur sehr dünne, wenig mehr als $50 \mathrm{~cm}$ hohe Halme und pflanzt sich durch unterirdische Ausläufer fort. Es ist wahrscheinlich eine Arundinaria-Art. Der echte Dendrocalamus strictus ist in Tausenden von Pflanzen aus Samen gezüchtet worden, die von Indien kamen.

\section{Palmen.}

Dem friiheren Mangel an Palmenarten in dem botanischen Garten ist in den letzten Jahren in sehr wirksamer Weise abgeholfen worden, so dass derselbe nunmehr eine recht stattliche Sammlung davon enthält. Die schon früher vorhanden gewesenen Corypha gebanga, Cocos eriospatha und Areca Catechu sind kräftig herangewachsen. Die letztgenannte Art ist durch Samen reichlich vermehrt worden und soll nunmehr zur Bepflanzung der Strassen von Viktoria mit Alleen benutzt werden.

Neu eingefuihrt sind durch die Zentralstelle folgende Arten, die fast alle gut gedeihen: Oreodoxa regia und O. oleracea, Phytelephas macrocarpa, die "Tagua" von Ecuador in zwei Varietäten, Carludovica palmata, Maximiliania regia, Astrocaryum rostratum 1. A. vulgare, Arenga saccharifera, Attalea cohune, Areca lutescens, A. glandiformis, Raphia taedigera, Stevensonia grandifolia, Martinezia caryotifolia, Cocos chilensis, Corypha elata, Calyptrogyne Swartzii, Caryota urens, C. Blancoi, Dictyosperma rubrum, Diplothemium canescens, Dypsis madagascariensis, Euterpe edulis, Hyphaene thebaica, Hydriastele Wendlandiana, Licuala spinosa, Livistona Hoogendorfi, L. subglobosa, Seaforthia elegans, Sabal Adansonii, Thrinax argentea, $T$. barbadensis und andere mehr.

\section{Zierpflanzen.}

Unter den Zierpflanzen sind besonders hervorzuheben 20 CodiaeumVarietäten mit bunten Blättern und 11 Varietäten von Hibiscus rosa sinensis mit verschiedenfarbigen Blüten, ferner Murraya exotica, Lagerstroemia indica und $L$. reginae, Acalypha tricolor und A. Wilkesiana, Panax dissectum und $P$. plumatum, Plumbago capensis und P. rosea, Beaumontia grandiflora, mehrere schönbluitige Bignonia-Arten, Petraea volubilis, Phyllanthus nivosus, Excoecaria bicolor, Bauhinia picta, Tabernaemontana jasminoides, Bougainvillea spectabilis, Dioclea lasiocarpa, Aristolochia cymbifera, Pincenectitia spectabilis, Eugenia densiflora, Jatropha podagrica, welche beiden letzteren bereits fruktifiziert haben und vermehrt worden sind, Musa Ensete, M. coccinea, Ravenala madagascariensis, Brownea coccinea und die prächtige Amherstia nobilis. 
Alle diese Zierpflanzen sind in der Umgebung des Direktorhauses, sowie in dem parkartig angelegten Teile des Gartens am Eingange und in der Nähe der Wege angepflanzt worden und beginnen bereits wesentlich zur Verschönerung des Gartens beizutragen. Eine grosse Anzahl ist auch in dem Schutzgebiete verbreitet worden.

An dieser Stelle eine Aufzäblung der eingesandten Sämereien zu geben, welche nicht gekeimt haben, oder derjenigen Pflanzen, welche bei dem Transporte nach Kamerun oder später in Viktoria verloren gegangen sind, wïrde wenig $Z$ weck haben und auch zu weit führen, obgleich die $Z$ ahl der letzteren verhältnismässig gering ist. $Z_{\text {war }}$ ist dieses oder jenes, wie schon erwähnt, fehlgeschlagen, im Durchschnitte muss man die erlangten Erfolge aber mindestens als zufriedenstellend bezeichnen, und der botanische Garten in Viktoria ist der botanischen Centralstelle in Berlin zu grossem Danke verpflichtet. Gerade die Einfuihrung neuer Arten aus fremden Weltteilen ist meist sehr schwierig, zeitraubend und kostspielig. Die Kultur und später die Vermehrung der Arten stösst bei der Gunst der Klima- und Boden-Verhältnisse in Viktoria schon auf geringere Schwierigkeiten, wenngleich es eines steten, miihsamen und leider oft genug erfolglosen Kampfes mit den zahlreichen, Vernichtung drohenden Schädlingen und unermïdlicher Aufmerksamkeit und Sorgfalt in der Pflege der Pflanzen bedarf. Das schnelle Wachstum der Pflanzen und die Genugthuung, welche man empfindet, wenn man in verhältnismässig kurzer Zeit die Frtichte und praktischen Erfolge seiner Arbeit sieht, entschädigt dann auch wieder reichlich für die aufgewendete Mühe und die gehabten Enttäuschungen.

\section{Notiz \\ zu dem Artikel, betreffend die Meliacee Pseudocedrela, in Nr. 28 des Notizblattes.}

Von

\section{H. Harms.}

Den Bemuhungen des Herrn Grafen von Zech ist es gelungen, nunmehr auch Blütenmaterial der Meliacee Pseudocedrela Kotschyi (Schweinf.) Harms im Togogebiete zu beschaffen. Es war die Vermutung ausgesprochen worden, dass diese Swietenioidee ein wertvolles, dem Mahagoni ähnliches Holz liefere, das vielleicht für den Export in Betraoht komme. Dazu bemerkt Herr Graf von Zech in einem aus Dyonayire vom 27. März 1902 datierten Briefe folgendes: „Nach meinem 